\title{
Reverse Logistics Network Design Based on Constraint Theory
}

\author{
Li Xiaohong \\ Jilin Province Economic Management Cadre College, Changchun, China
}

Guo Yushi

College of Economics and Management, China Jiliang University, Hangzhou, China

ABSTRACT: A reverse logistics network design model is proposed based on constraint theory. We can obtain the optimal solution including the number of facilities, locations and products flows for the network through the model. Genetic algorithms are developed to solve the model. Finally, a numerical example is shown to verify the validity of the model and the algorithms.

KEYWORD: reverse logistics network; constraint theory; network design; genetic algorithm

\section{INSTRUCTION}

Reverse logistics-related issues, such as product recycling, remanufacturing and reuse have attracted much attention. And other related fields from a customer recovery of used products, and then to convert it into reusable products or waste disposal all relevant logistics process [1]. The goal of product recovery is to recycle and use the residual value of used products [2]. These recycled products can become another resource to meet the demand for raw materials, then, instead of the traditional single vendor replenishment orders way. The size, cost and complexity of reverse logistics gradually changed the attitude of the manager in the regulations. In fact, the rational planning of reverse logistics can not only reduce costs, but also increase revenue. Reverse logistics system constituted by a plurality of links, reasonable logistics network design can help a company recover of materials and products with the minimal cost and maximal benefit. In respect of reverse logistics network optimization issues, some scholars have carried out some researches, Kroon and Vrijens[3] designed a returnable packaging recycling container logistics system and established a MILP (mixed integer linear programming model) to determine the number and location of the warehouse containers. Jayaraman[4] considered investment, transport, processing, and storage factors, MILP model is proposed to determine the recycling of electronic products remanufacturing location and quantity of plants. Le Blanc, Fleuren and Krikke [5] studied the car's gas tank recovery system, since about $50 \%$ of the total cost is transportation costs, and it is difficult to collect actual data, which the authors used the model to solve this problem Directions and used MILP models. MA Zujun [6], Zhao Yi [7], Demirel and Gokcen [8] and so on established a network design cost minimization deterministic MILP model. Therefore, the paper applies the theory of constraints thinking to construct reverse logistics network optimization design model, and gives numerical examples demonstrate the effectiveness of the model.

\section{THE REVERSE LOGISTICS NETWORK DESIGN MODEL}

\subsection{Model Preparation}

The theory of constraints (TOC) is a kind of management theory developed on the basis of optimized production technology (OPT) by the Israeli scientist Goldratt in 1980s, which is also a kind of decision making method. Its core idea is that the enterprise must put the limited resources and energy into the most important link, into the bottleneck of lowest logistics efficiency.

To construct of a reverse logistics network, including recycling centers, regional test center and reprocessing plant. The design of reverse logistics network in this paper is to determine the regional test center's number in the network, , the regional test center's location in the network, and the regional test center's the processing ability in the network, and the reasonable distribution logistics volumes in the constituting logistics path. In order to facilitate model, this paper makes the following assumptions: 
- Recovery processing related to a single returned products and single operation conditions in the model;

- According to the demand of regional total recovery product requirement, positions of the regional for demand are known;

- Recovery product demand, recycling of waste products recycling center, recovery of the quality of recycled products are independent random variables, obeying uniform distribution;

- The recycling center, the number of demand region and the position of each demand region are known;

- The unit transportation cost of each recycling products, the recycling center processing ability are known;

- The recovery rate can be controlled in a certain range, according to the actual needs to increase or decrease the amount of recovery.

For the convenience of narration, introduce the following notation:

Subscript:

$\alpha$ : Set up recycling center number, $\alpha \in\{1,2, \cdots, \mathrm{A}\}$.

$\beta$ : Set up testing center number, $\beta \in\{1,2, \cdots, B\}$.

$\lambda$ : The demand region number, $\lambda \in\{1,2, \cdots, A\}$.

The decision variables:

$y_{\alpha \beta}$ : The number of recycled products, the recycling center of $\alpha$ send to the testing center of $\beta$;

$y_{\beta \lambda}$ : The number of recycling products: regional test center $^{\beta}$ sent to manufacturers of $\lambda$;

$X_{\beta}$ : From the selected address whether $\beta$ as a regional test center, 0-1 variables;

$T_{\beta}$ : Design processing capacity of regional test center $\beta$.

Parameters:

$c_{\alpha \beta}$ : The transport costs of unit recycled products, recycling center $\alpha$ sent to the testing center $\beta$.

$c_{\beta \lambda}$ : The transport costs of unit recycled products, testing center $\beta$ send to the demand regional $\lambda$;

$v_{\beta}$ : The processing costs of unit recycled products in the testing center of $\beta$;

$f_{\beta}$ : Fixed cost of setting up regional test center $\beta$; $d_{\lambda}$ : Products manufacturer ${ }^{\lambda}$ on processed products demand;

$a_{\alpha}$ : Recycling of waste products recycling center $\alpha$ maximum;

$b_{\alpha}$ : Recycling of waste products recycling center $\alpha$ minimum;

$r_{\alpha}$ : Cost recovery of the waste products of unit is in the recycling center $\alpha$;

$P_{\lambda}$ : Acceptable price of units processed products in regional demand $\lambda$;

$M A X_{-} T_{\beta}:$ On recycling products available maximum detection capability in the testing center $\beta$;

$e$ : Recycled products can use ratio (i.e. rate of throughout).

\subsection{The Network Design Model}

Using the above parameters, decision model are as follows:

$$
\begin{aligned}
& \max E(T)=E\left(\sum_{\beta} \sum_{\lambda} y_{\beta \lambda} \cdot P_{\lambda}-\sum_{\alpha} \sum_{\beta} y_{\alpha \beta} \cdot\left(r_{\alpha}+c_{\alpha \beta}\right)\right) \\
& \min E(C)=E\left(\sum_{\beta} f_{\beta} \cdot X_{\beta}+\sum_{\beta} \sum_{\alpha} y_{\alpha \beta} \cdot v_{\beta}+\sum_{\alpha} \sum_{\beta} y_{\alpha \beta} \cdot c_{\alpha \beta}+\sum_{\beta} \sum_{\lambda} y_{\beta \lambda} \cdot c_{\beta \lambda}\right)
\end{aligned}
$$

s.t

$$
\begin{array}{lr}
b_{\alpha} \leq \sum_{\beta} y_{\alpha \beta} \leq a_{\alpha} & \forall \alpha \\
\sum_{\alpha} y_{\alpha \beta} \cdot e=\sum_{\lambda} y_{\beta \lambda} & \forall \beta \\
\sum_{\beta} y_{\beta \lambda}=d_{\lambda} & \forall \lambda \\
\sum_{\alpha} y_{\alpha \beta} \leq X_{\beta} \cdot T_{\beta} & \forall \beta \\
X_{\beta} \cdot T_{\beta} \leq M A X_{-} T_{\beta} & \forall \beta \\
y_{\alpha \beta}, y_{\beta \lambda}, T_{\beta} \geq 0 & \forall \alpha, \beta, \lambda \\
X_{\beta}=0 \text { or } 1 & \forall \beta
\end{array}
$$

The first objective of the model in (1) indicates to make the maximal effective output expectation value within planed period, the second objective of the model in (2) indicates that the minimal inventory and operation cost within the planning period. Constraint (3) denotes the number constraint of recovery products in the recycling center. Constraint (4) for the logistics balance constraints. Constraint (5) represents demand restriction. Constraint (6) is to 
ensure that recycling products of the number not exceeding the design capacity constraints in the input area of testing center. Constraint (7) represents each region detection center and design handling capacity which cannot exceed the maximum limit. Constraints (8) and (9) represent non negative constraint variables and 0-1 constraints.

\section{SOLVE METHOD FOR THE MODEL}

The above model is multiple objectives programming model, both objectives need to be optimized simultaneously, but two goals are not a simple compared, sometimes contradictory, therefore, this model does not necessarily exist to achieve the two objectives of the optimal solution, but there are a set of equilibrium solution, that is the so-called Pareto solutions, But because of many practical problems, the Pareto solution set of the general decision can be large, policymakers need to evaluate the solution set to choose from one of the best, and this work is often very complex. Therefore, this paper uses Gen and Cheng proposed compromise method based on distance measure, this method can obtain a compromise solution, not all solutions of Pareto.

\subsection{Algorithm overview}

- Code. The paper uses bit string coding.

- Selection. The roulette wheel selection method to select individual of the new population.

- Crossover. The paper uses a single point crossover.

- Mutation. This paper uses a single point mutation.

- Fitness function.

$\operatorname{Eval}(x)=P(x)\left(\frac{r_{\text {max }}-r(x)+e}{r_{\text {max }}-r_{\text {min }}+e}\right)$,

Among them $e \in(0,1), r(x)$ expresses regret value in the individual $\mathrm{x}, r_{\max }$ means current generation the biggest regret value, $r_{\min }$ means current generation the minimum regret value.

$$
P(x)=1-\frac{1}{\mathrm{~m}} \sum_{i}\left(\frac{\Delta b_{i}(x)}{\Delta b_{i}^{\max }}\right)^{a} .
$$

\subsection{The algorithm pseudo code}

In this paper, the genetic algorithm to solve the model pseudo code is as follows:

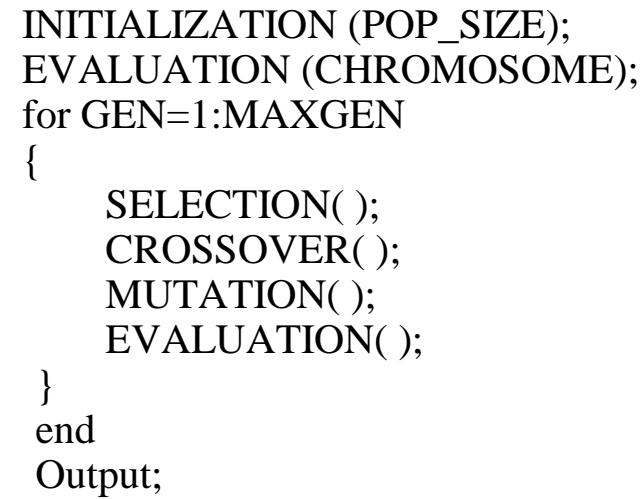

\section{NUMERICAL EXAMPLE}

$\mathrm{X}$ company is an automobile parts manufacturing enterprise, one of its branch is responsible for recovery processing of the parts, the processed parts can be sold to remanufacturing factories, suppliers of raw materials, secondary market. The branch has 3 products recycling centers, the existing 3 alternative addresses of pre-opened testing centers, and 3 demand regions. The demanding numbers of the 3 demand regions obey U[4 500, 5 300], $\gamma$ obeys the $\mathrm{U}[0.6,0.8]$, cost recovery PR for unit waste products is $¥ 10$ (assuming the recycling center all the same), acceptable price PM of units processed products is $¥ 30$ in demand region (assuming the demand for all regions are the same), minimum value of product recovery obeys $U$ [5 600, 6400$]$ in the recycling center, maximum values of the recovery volume obey U[7 800, 8 200], Processing cost of unit recovery product is $¥ 5$ in the testing center. In reality, the maximum detection capability may be different from each other in pre opened testing center, so we consider two situations: (1) The maximum detection capacity is the same in multiple testing centers, as 12000 , corresponding opened fixed cost is 15000 ; (2) Testing center 2 is $10 \%$ bigger than 1 , testing center 3 is $10 \%$ bigger than 2 , as 10800,12000 and 13200 respectively, opened fixed cost is 13500,15000 and 16 500.In order to compare, the total processing capacity and the total opened cost are the same in two situations. Other relevant data refer to in table 1-2. We have developed a genetic algorithm program with Matlab, simulation examples to test, According to the experience, genetic algorithm parameters: population size 600 to 500 , the evolution algebra, $\mathrm{p}=3$. The crossover rate, mutation rate were 0.8 and 0.1 , $W_{1}=0.6, W_{2}=0.4$ (The weight $W_{1}, W_{2}$ is assigned to each target). The best solution obtained sees in table 3 and table 4 . 
Table1.Transportation cost of unit recovery products sent to testing center from recovery center (yuan / piece).

\begin{tabular}{|c|c|c|c|}
\hline $\begin{array}{c}\text { recovery } \\
\text { center }\end{array}$ & $\begin{array}{c}\text { Alternative } \\
\text { address 1 }\end{array}$ & $\begin{array}{c}\text { Alternative } \\
\text { address 2 }\end{array}$ & $\begin{array}{c}\text { Alternative } \\
\text { address 3 }\end{array}$ \\
\hline 1 & 0.6 & 0.8 & 0.6 \\
\hline 2 & 0.9 & 1.3 & 0.5 \\
\hline 3 & 0.8 & 0.9 & 0.7 \\
\hline
\end{tabular}

Table2.Transportation cost of the unit products sent to demand region from testing center (yuan / piece).

\begin{tabular}{|c|c|c|c|}
\hline $\begin{array}{c}\text { Alternative } \\
\text { address }\end{array}$ & $\begin{array}{c}\text { Demand for } \\
\text { regional 1 }\end{array}$ & $\begin{array}{c}\text { Demand for } \\
\text { regional 2 }\end{array}$ & $\begin{array}{c}\text { Demand for } \\
\text { regional 3 }\end{array}$ \\
\hline 1 & 0.9 & 0.8 & 1 \\
\hline 2 & 1.2 & 1.1 & 1.3 \\
\hline 3 & 0.7 & 0.8 & 0.7 \\
\hline
\end{tabular}

Table3.The best scheme of case 1

\begin{tabular}{|c|c|c|}
\hline Alternative address & 1 & 3 \\
\hline$T_{\beta}$ & 11522 & 12000 \\
\hline$y_{1 \beta}$ & 4802 & 3198 \\
\hline$y_{2 \beta}$ & 1348 & 5952 \\
\hline$y_{3 \beta}$ & 2850 & 2849 \\
\hline$y_{\beta 1}$ & 2100 & 2800 \\
\hline$y_{\beta 2}$ & 2100 & 2800 \\
\hline$y_{\beta 3}$ & 2100 & 2800 \\
\hline
\end{tabular}

Table4. The best scheme of case 2

\begin{tabular}{|c|c|c|}
\hline Alternative address & 1 & 3 \\
\hline$T_{\beta}$ & 9000 & 12000 \\
\hline$y_{1 \beta}$ & 8000 & 0 \\
\hline$y_{2 \beta}$ & 0 & 8000 \\
\hline$y_{3 \beta}$ & 1000 & 4000 \\
\hline$y_{\beta 1}$ & 2050 & 2850 \\
\hline$y_{\beta 2}$ & 2107 & 2793 \\
\hline$y_{\beta 3}$ & 2143 & 2757 \\
\hline
\end{tabular}

In two situations, to construct regional test center in 1 and 3 alternative address, and according to the calculation results in the table, to distribute logistics volume of recover products in the reverse logistics network. At the same time, in situation 1, the effective output is 21770yuan, inventory and operation cost is 16000yuan, in situation 2, the effective output is 21860yuan, inventory and operation cost is 15923yuan. Visible situation 2 objective optimization result is much better than the optimal result of situation 1 .

\section{CONCLUSION}

In order to meet the demand of different enterprises, this paper designs the general recycling logistics network model. At the same time, the theory of constraints is extended, constructing an optimization model based on constraints theory in the reverse logistics network, and using the real condition of enterprise data to verify the validity of the model. The system increases effective output, and reduces inventory and operating costs correspondingly, to improve the competitiveness of enterprises, to further strengthen the scientific management of recycling logistics system, and to promote environment-friendly development model of the establishment, also to obtain more sales revenue. It provides reference to the actual decision-making of enterprises. According to the actual demands, considering much more uncertainty, an integration model of multi cycles can be constructed.

\section{ACKNOWLEDGEMENT}

The research of the authors was supported, in part, by National Nature Science Foundation of China (no. 71402173), Science Foundation of Ministry of Education of China (no. 14YJC630168), and Hangzhou Science and Technology Information Research Project (no. 20131334M10).

\section{REFERENCES}

[1] Minner S, Kleber R. 2001. Optimal control of production and remanufacturing ina simple recoverymodel with linear cost functions. OR Spektrum: (23):3- 24.

[2] Beullens P. 2004.Reverse logistics in effective recovery of products from waste materials. Reviews in Environmental Science \&Bio/Technology: (3):283- 306.

[3] Kroon L,Vrijens G. 1995. Returnable containers: an example of reverse logistics. International Journal of Physical Distribution \&Logistics Management: 25(2):5668.

[4] Jayaraman V, Raymond A, Erik Rolland. 2003. The Design of Reverse Distribution Networks Models and Solution Procedures. European Journal of Operational Research: 150(1):128- 149.

[5] Blanc H M, Fleuren H A, Krikke H R. 2004. Redesign of a recycling system for LPG- tanks. OR Spectrum: (26):283- 304.

[6] Ma Zujun, Dai Ying. 2005. Optimal design model of reverse logistics network for product recovery. Journal of management in Engineering: 19 (4): 114- 117.

[7] Zhao Yi, Yin Chuanzhong, Pu Yun. 2005. The recovery logistics facilities of multilayer location model and algorithm. Journal of Southwest Jiao Tong University: 40 (4): 530- 534

[8] Demirel No, Gokcen H. 2007.A mixed integer programming model for remanufacturing in reverse logistics environment. Int J Adv Manuf Technol. 